\title{
Trust me, I'm a dentist
}

\author{
R. Matthews
}

\section{This is the fifth article in a series written by a selection of experts who will be speaking at this year's National Dental Conference: Best Practice 2001. In this issue, Roger Matthews 1 explores the issue of trust between patient and dentist and the ways in which the profession can improve the quality of its service.}

$\mathrm{M}$ y apologies to Dr. Rob Buckman, then a health columnist at the Independent, for plagiarising his title'Trust me I'm a doctor', when I presented a short paper on the occasion of the first Demos Report 'Open Wide Futures for Dentistry' in 1996.

The title struck a chord - since then it has been adopted by some of the most widely read dental writers. However, there still remain unanswered the questions inherent in the title:

Patient: 'But why should I trust you?'

Dentist: 'But why should you not trust me?'

It is only a few short years since science and the professions appeared to be triumphant. Magic bullet therapeutics, NASA space missions and a seemingly unlimited potential for a bright technological future in which increased life-expectancy and quality of health care were unquestioned attributes.

And now? After Three Mile Island and Piper Alpha? After the Bristol paediatric cardiac incident and Dr. Shipman? After Digging For Gold and continuing high-profile dental malpractice incidents?

AIDS and BSE may seem a million miles from the dental profession's daily endeavours on behalf of patients, but to a public informed by tabloid media and vigilante consumerism, they can all too easily appear part of a continuum of scientific blunders and professional self-interest.

We recognise that the man and woman in the street still 'trust' their own dentist (assuming they are one of the 59 per cent who claim to attend for regular examinations). Indeed, the BDA's own research has re-emphasised this fact. Additionally, where public opinion is sampled on the comparative trustworthiness of professions, dentists still rank comparatively highly, alongside

${ }^{1}$ Roger Matthews is Chief Dental Officer for Denplan Limited. pharmacists, vets and, yes, family doctors.

However, overall trust in professions generally, and health professions in particular, has declined in the past quarter century. Professional self-regulation has come under increasing threat in those parts of the world where it is still a reality and there is no better exemplification of the change in ethical values than the current re-examination of parental consent to medical procedures for children following the Alder Hey Hospital revelations.

There is no-one to look to, outside the professions themselves, for a sea change in this decline. So long as 'bad news is headline news', we look in vain for support from media, governmental or consumerist interests.

The procedures and attitudes which we and our supporting teams employ in dealing with each patient are the sole measure by which we can counter this threat. The critical means we can employ include, but are not limited to:

- True patient involvement in care, flowing from an appreciation of patient autonomy

- Communication and consent in terms which the patient can appreciate and understand

- A recognition that there are almost always therapeutic alternatives and that all outcomes are uncertain

- A continual striving to improve and develop our skills and an openness to constructive appraisal

- The abolition of a blame culture within the profession, linked to a intra-professional awareness of the superior rights of patients when they are perceived objectively to be at risk

Patient involvement in care - the 'shared care' concept is an area where dentists might quite legitimately claim to have made many advances. The patient who claims to want no participation and to leave all decisions to the dentist is still commonplace, but increasingly, we wisely explain none the less and ensure that the notes are clear and comprehensive.

Communication is a talent which most dentists will claim to possess in full measure, but objective analysis tends to expose our weaknesses and the natural tendency for jargon and even the tendency to look down on 'plain English' does not help us.

The recognition that treatment alternatives exist and that the patient has the right to choose (and to choose not to have treatment at all) is an issue in an age of increasing specialisation. 'When you are a hammer', goes the saying, 'everything looks like a nail'.

The advent of clinical governance will place upon all health professionals a statutory obligation to demonstrate the presence of a quality assurance programme with the object of continually improving our patient care. The challenge is to find and employ such a programme which is clear, achievable and relevant to our role.

Finally, as a profession we need to look at our own perception of ourselves and our colleagues. The concept of 'whistleblowing' has been with us for some time, but the practical implications of this policy have yet to be more widely researched in dentistry.

In a changing world, there is, of course, the temptation to simply carry on as before: there is, of course, no single law which requires any of us to pursue the objectives considered here.

The words of J. Edwards Deming were, however, well chosen: 'Change is not essential, for survival is not mandatory?.

The Best Practice 200I

Conference will take place

between 3-5 May 200 I at the

Harrogate International Centre.

For a copy of the registration

form please contact the Events

Office on 02075634590. 